$\mathbf{X}$.

\title{
Weitere Mittheilungen über die Innervation des M. crico-thyreoideus.
}

\author{
Von Dr. J. Katzenstein in Berlin.
}

In einer früheren Arbeit ${ }^{1}$ ) erbrachte ich den Nachweis, dass ein N. laryng. med. im Sinne Exner's nicht existire. Ich schloss dieselbe mit den Worten: „Der betreffende Nerv ist beim Hunde der N. pharyng. med. e vago, beim Affen der N. pharyng. med. e vago et sympathico". Für das Kaninchen hatte ich diesen Beweis nicht geführt.

Herr Prof. Exner demonstrirte nun in der Sitzung der physiologischen Gesellschaft zu Berlin vom 9. December 1892, dass eine Contraction des M. crico-thyreoid. beim Kaninchen erfolgt, wenn man den $\mathrm{N}$. pharyng. med. e vago reizt.

Der Referent über die Sitzungen der physiologischen Gesellschaft schreibt darüber ${ }^{2}$ ) Folgendes:

"Herr Sigmund Exner (Wien, als Gast) demonstrirt den Eriolg, welchen die elektrische Reizung des von ihm vor Jahren entdeckten N. laryng. med. beim Kaninchen hat. Durch Exstirpation der beiden submaxillaren Speicheldrüsen waren die tieferen Gewebslagen der oberen Keblgegend ausgiebig freigelegt und der Kehlkopf so um seine Längsaxe gedreht, dass die eine Seite desselben mit dem M. crico-thyreoid. und mit der Eintrittsstelle des N. laryng. sup. frei vorlag. Dieser Nerv war in seiner ganzen Länge präparirt, nahe an seinem Ursprunge aus dem Vagus durchschnitten und so angeschlnngen, dass sein peripherischer Stumpf an dem Faden aus der Wunde gehoben werden konnte. Ebenso war der Laryng. med. bei seinem Austritte aus dem $R$. pharyng. vagi durehschnitten und so angeschlungen, dass er aus der Wunde geboben und ihm in der Luft ein mit der Hand geführtes Platin-Elektrodenpaar angelegt werden konnte. Das Elektrodenpaar war mit der secundären Spirale eines Schlitteninductoriums verbunden und bei vorsichtiger Reizung jedes einzelnen dieser Nerven contrahirte sich der vorliegende M. crico-tbyreoid. in ausgiebiger Weise, was sowohl an der Form-

1) Katzenstein, Ueber die Innerv. d. M. crico-thyreoid. Dieses Archiv. Bd. 130.

2) Deutsche med. Wochenschr, 1893. No, 6. 
veränderung des Muskels, als an der einseitigen Annäberung der Cartilago cricoidea an die Cartil. thyreoidea sebr deutlich zu sehen war. Exner erläuterte die speciellen anatomischen Verbältnisse, wie sie, etwas abweichend beim Kaninehen and beim Hunde liegen und sagte, dass er dem Kaninchen für diese Demonstration aus zwei Gründen den Vorzug gegeben habe. Erstens seien ibm seine Angaben über den Laryngeus medius beim Hunde schon von anderer Seite bestätigt worden, beim Kaninchen aber noch nicht, und zweitens sei die Beweisführung beim Hunde in der That nicht so leicht, wäbrend die Demonstration beim Kaninchen so sicher gelinge, dass sie zu seinen regelmässigen Vorlesungsexperimenten gehöre."

In dem Berichte, den Exner selbst über diese Demonstration $\mathrm{gab}^{1}$ ), bemerkt er, dass er das Kaninchen zur Demonstration wählte, weil bei diesem Thier der $\mathrm{N}$. laryng. med. noch nicht von anderer Seite bestätigt worden sei und weil der Versuch am Kaninchen weit leichter und sicherer auszuführen sei als beim Hunde. Exner sagt nun wörtlich Folgendes:

„Da Katzenstein diesen Nerven für beide Thiere, an denen ich experimentirt hatte, für Hund und Kaninchen läugnete, so war es bei Erweisung meiner Behauptung gleichgültig, an welches der Thiere ich mich hielt. Ein Zwischenruf in der Discussion, die sich an meinen Vortrag knüpfte, sollte mich darüber belehren, dass Katzenstein seine Abweisung des Nerven für das Kaninchen nur anf die anatomische Verfolgung und auf Degenerationsversuche, nicht auf Reizungsversuche gegründet babe, was ich allerdings schon aus seiner Abhandlung vermuthet batte."

Den betreffenden Zwischenruf habe ich nicht gemacht. Am Schlusse meiner ersten Abhandlung habe ich ausdrücklich bemerkt a. a. O. S. 330: Der betreffende Nerv ist beim Hunde der N. pharyng. med. e vago, beim Affen der N. pharyng. med. e vago et sympathico. Demnach hebe ich hervor, dass ich mich bezüglich des Kaninchens jeder Aeusserung enthalten habe. Der obige Ausspruch Exner's, dass ich den N. laryng. med. für das Kaninchen geleugnet habe, ist demnach nicht zu Rechte bestehend.

Exner bemerkt ferner, dass seine Untersuchungen für den Hund in allen wesentlichen Punkten von Onodi, Livon, Ellenberger und Baum bestätigt worden seien. Es verlohnt, die Ausführungen dieser Forscher hier nochmals anzuführen, um die Gegensätze zu den Darlegungen Exner's daraus zu erweisen:

1) Exner, Die Innerv. d. M. crico-thyreoid. Dieses Archiv. Bd. 131. 
On odi ${ }^{1}$ sagt: „Die Untersuchungen haben gezeigt, dass der von $\mathrm{Exner}$ aufgestellte N. laryng. med. in seiner morphologisehen Erscheinung nicht jene Selbständigkeit besitzt, welche von Seite $\mathrm{Exu}$ ur's behauptet wurde. Ich habe aus mehreren Gründen vorgeschlagen, einfach von Verbindungen $z u$ sprechen, welche $z$ wischen dem R. pharyng. vagi und dem äussersten Zweige des Laryng. sup. bestehen."

In einer jüngst erfolgten Publication ${ }^{2}$ ) führt Onodi ausserdem Folgendes aus: „Bevor wir die von Exner anfgestellte Lehre über die doppelte Innervation der Kehlkopfmusculatur besprechen, müssen wir uns mit der Frage des N. laryng. med. befassen. Den Namen, sowie die Deutung seiner Bedeutung verdanken wir ebenfalls Exner. Mit der Bezeichnung N. laryng. med. bat Exner einen $Z$ weig des R. pharyng. vagi beschrieben, welcher zum M. crico-thyreoid. geht Dieser Nerv wird beim Kaninchen und Hunde als selbständiger Nerv beschrieben, beim Menschen war Exner bestrebt, ein Homologon zu suchen.

Gegen seine Existenz haben sich auf Grund physiologischer Versuche Simanowsky ${ }^{3}$ ) und Katzenstein ${ }^{4}$ ) erklärt. In früheren Mittheilungen habe ich mich sowohl über die morphologische, wie auch über die physiologische Bedeutung des $\mathbf{N}$. laryng. med. erklärt. Ungeachtet seiner physiologischen Bedeutung, dessen Kenntniss wir Exner verdanken können, spielt der N. laryng. med. in seiner morphologischen Bedeutung eine untergeordnete Rolle. Er ist beim Kaninchen selbständig zu treffen, bei Hunden habe ich ihn in Form einer Verbindung zwisehen dem R. pharyng. vagi und R. ext. n. laryng. sup. getroffen, beim Menschen gar nicht getroffen.

Ich habe vorgeschlagen, anstat dem Namen Laryng. med. einfach von Communicanten zu sprechen oder von Fäden, welche selbständig oder in Form einer Verbindung beim Kaninchen und Hunde ausser der Bahn des R. ext. n. laryng. sup. zum M. crico-thyreoid. ziehen. Ich habe mich experimentell überzeugt, dass bei diesen diese Fasergattung mit der Innervation des M. crico-thyreoid. verbunden ist." Ich habe die sichtbaren Contractionen des Muskels graphisch aufgenommen, weiterhin habe ich bei einem Hunde diese Faserbündel exstirpirt und das Thier nach zwei Monaten getödtet, die Mm. crico-thyreoid. waren stark degenerirt.

Gegen Simanowsky und Katzenstein muss also constatirt werden, dass diese von Exner erkannten Faserbündel an der Innervation des M. crico-thyreoid. einen directen Antheil baben. In morphologischer Hinsicht, da sie beim Menschen fehlen, betrachte ich diese Faserbündel als einen ausgeschalteten Theil der sonst im Laryng. sup. enthaltenen motorischen Fasern und bin daher gegen die Bezeichnung: Laryngeus medius."

1) Onodi, Berl. klin. Wochensehr. 1893. No. 10.

2) Onodi, Monatsschr. f. Ohrenheilk. 1887. Centralbl. f. d. med. Wissensch. 1888.

3) Simanowsky, Arch. f. Phys. Bd. 42/43.

4) a. a. 0 . 
Den letzten Bemerkungen gegenüber muss ich betonen, dass ich diese Verbindung des N. pharyng. med. mit dem äusseren Aste des N. laryng. sup. ${ }^{\text {I) }}$ bescbrieben habe, in dem ich sagte: In seltenen Fällen war sowohl beim Hunde wie beim Affen eine Anastomose zwischen dem R. ext. des N. laryng. sup. und dem N. pharyng. med. - dem N. laryng. med. Exner's - zu beobachten.

Ganz im Gegensatze zu Exner findet Onodi an dem M. crico-thyreoid. des Hundes, dem er den N. pharyng. med. exstirpirt und den er nach zwei Monaten getödtet hat, „die Mm. crico-thyreoid. stark degenerirt“. Exner ${ }^{2}$ ) sagt dagegen: „Die Durchtrennung des N. laryng. med. macht keine Degeneration des gleichseitigen M. erico-thyreoid.; dieselbe tritt erst ein, wenn der gleichseitige N. laryng. sup. und med. derselben Seite durchsebnitten werden". An anderer Stelle äussert Exner im Gegensatz zu Onodi genau dasselbe: „Wenn zwei Nerven mit einem Muskel derart in Verbindung stehen, dass die elektrische Reizung zwei der ersteren den letzteren zur Contraction bringt und man durchschneidet einen Nerven und lässt das Thier am Leben, so kann man Wochen oder Monate vergebens nach degenerirten Muskelfasern in dem betreffenden Muskel suchen. Erst wenn beide Nerven durchschnitten werden, degenerirt der Muskel.

Die Angabe Onodi's, dass er nach Exstirpation des N. pharyng. med. eine starke Degeneration in dem gleichseitigen M. crico-thyreoid. gefunden habe, ist zu berichtigen, da meine Degenerationsversuche am Kaninchen und am Hunde gezeigt haben, dass

1) an normalen Muskeln des Kehlkopfes an einem grossen Theile der Muskelfasern Veränderungen wie Schwund der Querstreifung, körnige Auflagerungen an jedem Präparate nachzuweisen sind;

2) die Durchschneidung des $N$, pharyng. med. e vago eine beträchtliche, aber keine vollständige Atrophie der gleichseitigen Pharynxmusculatur zur Folge hat und dass der gleichseitige M. crico-thyreoid. sich wie ein normaler verhält.

Nach Ellenberger und $B a m^{2}$ ) giebt der „R. pharyng. sup. (ant.) an den N. laryng. sup. einen Verbindungszweig; diesen nennen sie nach Onodi N. laryng. med. Die Erläuterung zu Fig. 184 ihres Werkes lautet jedoch: „f. R. pharyng. sup. vagi; g. dessen R. anastomoticus (laryng. med.?) zum N. laryng. sup.". Aus dem Fragezeichen geht hervor, dass

a) a. a. O. Dieses Archiv. Bd. 130. S. 322 .

2) Ellenberger und Baum, Anatomie des Hundes. 
Ellenberger und Baum entweder einen N. laryng, med, überhaupt oder im Sinne Onodi's in Frage stellen.

Nach Livon I) enthielt der M. crico-thyreoid. accessorisebe Fasern von einem kleinen, aus dem Pl. pharyng. stammenden Nerven, der sich mit dem R. ext. motorius des N. laryng. sup. kurz vor dem Eintritt des letzteren in den M. crico-tbyreoid. verbindet.

Nach diesen Darlegungen muss ich meinen früher aufgestellten Satz: Es haben somit nur Exner und seine Schüler Wagner und Réthi den N. laryng. med. übereinstimmend beschrieben, alle anderen Autoren sind zu mehr oder minder von einem der genannten Autoren abweichenden Resultaten gekommen, - aufrecht erhalten.

Ich habe es bei dieser Lage der Dinge für nöthig erachtet, der Frage: Innervirt der N. pharyng. med. e vago den M. cricothyreoid. oder nicht, noch einmal nahe za treten.

Erwähnen will ich, dass in der Anatomie des Kaninchens von Schneider ${ }^{2}$ ) folgende Angabe steht: "Bevor noch der Vagus seinen Pl. ganglioformis bildet, geht von ihm ein zartes Nervenästchen, R. pharyng. vagi ab, welches etwa in gleicher Höhe mit dem grossen Zungenbeinhorn medianwärts läuft und mehrfach gespalten zwischen die Fleischbündel des mittleren Schlundschnürers sich einsenkt."

W. Krause ${ }^{3}$ ) erwähnt nur: „In der Höhe der Theilungsstelle der A. carotis com. entspringt der N. laryng. sup., sowie der R. cardiacus, etwas oberhalb die Rr. cardiaci.

In den nachfolgenden Untersuchungen wurden der anatomische Verlauf des N. pharyng. med. festgestellt, sowie die Resultate, die sich bei elektrischen Reizungen des Nerven ergaben. Als Versuchsthiere dienten Kaninchen, Hunde, Katzen, Affen. Bei letzteren zwei Thierspecies sind elektrische Reizungen des N. pharyng. med. e vago bisher nicht ausgeführt. Die Versuche wurden mit gütiger Erlaubniss des Herrn Prof. H. Munk in dem physiologischen Laboratorium desselben vorgenommen. Herr Prof. Munk stand mir, so oft es Noth that, mit seinem be-

1) Livon, Travaux de Physiol. expérim. 1890/91. p. 131.

2) Schneider, Top. Anat. des Yorderhalses beim Kaninchen. S. 12.

3) W. Krause, Die Anatomie des Kaninchens in topogr. u. operat. Rücksicht. S. 316. 
währten Rathe bei und sage ich ibm dafür meinen herzlichsten Dank.

Bei neun Kaninchen wurde Folgendes gefuuden: Vom N. vagus geht im For. jugulare, oft zwischen diesem und dem $\mathrm{Pl}$. nodosus der $\mathrm{N}$, pharyng. vagi ab, anastomosirt mit dem $\mathrm{N}$. glossopharyng., läuft demselben parallel nach vorn und abwärts und theilt sich alsbald in zwei $Z$ weige. Der obere Ast senkt sich in die Hinterwand des Pharynx; der untere Ast läuft hinter dem Pharynx nach abwärts, wobei er wenige mm medianwärts von dessen Seitenrande liegt, bohrt sich in der Höhe des oberen Kehlkopfrandes angelangt in den $M$. thyreo-pharyng. neben dem Ursprung des letzteren am Kehlkopfe ein, kreuzt in demselben den N. laryng. sup., der aussen diesem Muskel anliegt und endigt in ihm. Ein Uebergang in den M. crico-thyreoid. bestand nicht.

Zam Zwecke der elektrischen Reizung wurden die Kaninchen mit Aether narkotisirt. Der N. pharyng. med. wurde freipräparirt und unterbunden. Der Strom wurde von einem $\mathrm{du}$ Bois-Reymond'schen Inductorium mit Bunsen'schem Tauchelement geliefert.

Es ergab sich bei allen Reizungen am N. pharyng. med. des Kaninchens, dass durch dieselbe eine Contraction im Gebiete des gleichseitigen M. crico-thyreoid. ausgelöst wurde. Bei Reizung des N. pharyng. med. waren stets schwächere Stromstärken nothwendig, um eine Contraction des M. crico-thyreoid. hervorzubringen als beim N. laryng. sup., bezügl. bei gleichen Stromstärken war die Contraction im M. crico-thyreoid. stärker bei Reizung des N. pharyng. med. als bei Reizung des N. laryng. med. Des Ferneren contrahirten sich bei Reizung des N. pharyng. med. der Oesophagus, so weit man ihn überblicken kann, und die gleichseitige Pharynxmusculatur, Die Contraction beginnt stets am Oesophagus unten, ergreift dann die hintere Pharynxwand und giebt sich gleichzeitig am M. crico-thyreoid, kund. Es findet eine sehr starke Annäherung der Cart. cricoid. an die Cart. thyreoid. dabei statt.

Demnach steht fest, dass in der von Exner angegebenen Weise der M. crico-thyreoid. beim Kaninchen eine doppelte Innervation hat und zwar durch den $\mathrm{N}$. laryng. sup. und den N. pharyng. med. 
Die anatomische Präparation des N. pharyng. med. beim Hunde deckte sich genau mit meinen früher gefundenen Ergebnissen. Zwischen dem Pl. nodosus und dem For. jugulare, oft auch im For. jugulare entspringt aus dem Vagus der N. pharyng. vagi. Derselbe giebt eine Anastomose zum N. glossopharyng. und theilt sich dann in drei Zweige. Der oberste verläuft nach vorn in der Höhe der Membr. thyreo-hyoid. und geht alsbald in die Pharynxmusculatur, in welcher er verschwindet. Der zweite Ast läuft abwärts, liegt am äusseren Rande des M. thyreopharyng., kreuzt den inneren Ast des N. laryng. sup., an dessen medialer Seite er liegt, und geht alsdann eine kurze Strecke parallel dem äusseren Aste des N. laryng. sup., wendet sich dann nach hinten und abwärts, um im M. constrictor pharyng. inf. zu verschwinden; in diesem theilt er sich in einen schwächeren oberen und einen stärkeren unteren Ast. Ein Uebergang des zweiten Astes in den M. crico-thyreoid. bestand nicht. Der dritte Ast läuft parallel dem N. pharyng. med. nach abwärts, kreuzt den inneren und äusseren Ast des N. laryng. sup., unter denen er liegt und tritt in den M. cricopharyng. ein.

In mehreren Fällen war eine Anastomose zwischen dem R. ext. des N. laryng. sup. und dem N. pharyng. med. zu beobachten.

Die elektrische Reizung des N. pharyng. med. hatte in 15 Fällen folgende Resultate:-

21. Februar 1893. Spitzhündin. Freilegung des ganzen N. laryng. sup. sin.; desgleichen des N. pharyng. med. sin. Letzterer entspringt böher, als der N. laryng. sup. ans dem Vagus und zwar erst im For. jugulare. Exstirpation des ganzen N. laryng. sup. Bei Reizung des N. pharyng. in der Nähe seines Ursprunges contrahirt sich von $350 \mathrm{~mm}$ Rollenabstand an bis zu sehr starken Strömen nur die Pharynxmusculatur und zwar so, dass dieselbe nach unten gezogen wird; zugleich wird der ganze Larynx und wit ihm der M. crico-thyreoid. nach links heruntergezogen. Der Oesophagus contrahirt sich, so weit sich derselbe übersehen lässt.

23. März. Hund. Freilegung des ganzen N. pharyng. med. und des N. laryng. sup. links. Reizung des N. pharyng. med. mit schwächsten Strömen: Die gesammte Pharynxmusculatur contrahirt sich; dabei wird der M. crico-thyreoid. sin. in toto nach links und unten heruntergezogen, contrahirt sich aber nicht. Dagegen bildet sich bei Reizung des N. pharyng. med. dadurch, dass der Muskel herabgezogen wird, zwischen den beiden 
Partien des M. crico-thyreoid. eine deutliche Falte, die jedoch keine Contraction ist. Diese Fältelung wird in allen Fällen dadurch hervorgerufen, dass durch die Contraction des M. constrictor pharyng. inf. eine Verschiebung der lateralsten Partien des M. crico-thyreoid. erzeugt wird. Genau dasselbe erfolgt, wenn man den M. constrictor pharyng. inf. reizt. - Die Reizung des N. laryng. sup. ruft typische Contraction des M. crico-thyreoid. hervor; dabei zieht sich der ganze Muskel von rechts unten nach links oben, wobei die Cart. cricoid., da sie nur einseitig ewporgezogen wird, scbief nach oben gedreht wird.

27. März. Hund. Freilegung des N. pharyng. und N. laryng. sup. sin. Reizung des N. pharyng. med. ergiebt eine kräftige Contraction der ganzen Pbarynxmusculatur, sowie ein Herüberziehen des M. crico-tbyreoid. auf die linke Seite und nach unten. Der M. crico-thyreoid. zeigt dabei in der Mitte eine Falte, jedoch keine Contraction. - Section der rechten Seite. Der $N$. pharyng. vagi theilt sich in die bekannten drei $Z$ weige, von denen der mittlere in die Pharynxmusculatur wie gewöhnlich eingeht. Ein Uebergang in den M. crico-thyreoid. ist nicht zu constatiren, desgleichen besteht keine Anastomose mit dem N. laryng. sup.

20. Juli. Sehr grosser Jagdhund. Freilegung des linken N. vagus, der linken $N$. laryng. sup. und $N$. pharyng. med. Es besteht zwischen dem äusseren Aste des N. laryng. sup. und dem N. pharyng. med. eine Anastomose. Ein Cebergang des N. pharyng. med. in den M. crico-thyreoid. findet nicht statt. Bei Reizung des äusseren Astes des N. laryng. sup. contrahirt sich der M. crico-thyreoid. vollständig. Reizung des N. pbaryng. med. oberbalb der Anastomose $z$ wisehen Laryng. sup. und Pharyng. med. ruft gleichfalls eine Contraction des gesammten M. crico-thyreoid. hervor. Durchschneidet man jetzt die Anastomose und reizt den N. pharyng. med, so findet nur noch eine Fältelung des M. crico-thyreoid. in seinen lateralsten Partien statt. Es wurde zuletzt der ganze N. laryng. sup. exstirpirt. Reizt man nun den N. pharyng. med., so war Fältelung des M. crico-thyreoid. kaum noch wahrzunehmen. Der Muskel wird nach unten und nach der linken Seite gezogen.

26. Juli. Hund. Freilegung des linken N. pharyng. med. und. N. laryng. sup. Bei Reizung des N. pharyng. med. bemerkt man an der lateralen Partie des M. crico-thyreoid. eine ganz geringfügige Fältelung, die jedoch keine Contraction ist. Dieselbe Fältelung tritt bei Reizung des M. constrictor pharyng. inf. genau in gleicher Weise auf.

Die übrigen vorgenommenen 10 Versuche am Hunde stimmten je mit einem der vorhergehend geschilderten überein. Ich führe deshalb die Protocolle hier nicht aus.

Es wurden fernerhin Controlversuche am Hunde folgendermaassen angestellt: Der N. vagus wurde bis zu seinem Eintritte in das For. jugulare freipräparirt, desgleichen der N. laryng. sup. und der N. pharyng. vagi bis zu ihrem Eintritte in den Vagus. 
Alsdann wurde der $N$. vagus so weit wie möglich oberhalb der Abgangsstelle des $\mathrm{N}$. pharyng. vagi unterbunden und abgetrennt. Der N. laryng. sup. wurde exstirpirt. Reizt man dann den $\mathrm{N}$. vagus oberhalb der Abgangsstelle des $\mathrm{N}$. pharyng. vagi, so müsste, falls eine Verbindung des N. pharyng. med. mit dem M. cricothyreoid. besteht, eine Contraction des letzteren erfolgen. Es findet jedoch nur eine sehr starke Contraction der gleichseitigen Pharynxmusculatur statt; die Cart. cricoid. wird dabei nach abwärts gezogen und der M. crico-thyreoid. der Länge und dem Verlaufe seiner Fasern nach gedehnt. Eine Contraction des M. crico-thyreoid. findet nicht statt. Reizt man den N. pharyng. med. nach seinem Abgange ans dem Vagus allein, so wird der M. crico-thyreoid, wie bei den vorhergehenden Versuchen nach unten und nach der Seite des betreffenden Nerven durch den Zug der sich contrahirenden Pharynxmusculatur gezogen.

3. Juli. Hund. Freilegung des linken N. vagus, des linken N. pharyng. med. und des linken N. laryng. sup. Exstipation des linken N. laryng. sup. Abtrennung und Cnterbindung des $\mathrm{N}$. vagus aberhalb der Abgangsstelle des $\mathrm{N}$. pharyng. vagi. Reizung des $\mathrm{N}$. vagus oberbalb der Abgangsstelle des $\mathrm{N}$. pharyng. vagi: Der M. crico-thyreoid. contrahirt sich dabei nicht, sondern er dehnt sich der Länge nach, und zwar wird dies dadurch erzeugt, dass die Cart. cricoid. durch die ganze in Contraction versetzte Pharynxmusculatur nacb abwärts gezogen wird. Reizt man den N. pharyng. med. für sich, so contrahirt sich die zugehörige Pharynxmusculatur; der ganze Kehlkopf und mit ihm der M. crico-thyreoid. werden nach links und unten gedreht; eine Contraction ist an ihm nicht zu bemerken.

8. Juli. Hund. Freilegung des linken N. vagus, des N. laryng. sup. und des $\mathrm{N}$. pharyng. med. Exstirpation des ganzen N. laryng. sup. Abtrennung und Unterbindung des $\mathrm{N}$. vagus oberbalb der Abgangsstelle des N. pharyng. vagi. Reizung des N, ragus daselbst. Die Cart. cricoid. wird nach abwärts gezogen und in Folge dessen der M. crico-thyreoid. der gleichen Seite gestreckt. Reizt man den N. pharyng. med. sin, allein, so wird der Larynx nach links und unten heruntergezogen, eine Contraction des M. crico-thyreoid. erfolgt nicht.

12. Juli. Hund. Freilegung des linken N. vagus, N. laryng. sup., N. pharyng. vagi. Exstirpation des N. laryng. sup. Reizung des N. vagus oberhalb der Abgangsstelle des N. pharyng. vagi. Die Cart. cricoid. wird dabei nach nnten gezogen und der M. crico-thyreoid. seiner Länge und seinem Faserverlaufe nach gedebnt. Bei Reizung des N. pharyng. med. allein wird der M. crico-thyreoid. nach links und unten gedreht, aber nicht contrahirt; dagegen contrabiren sich die zugehörigen Pharynxmuskeln und der Oesophagus, so weit man ihn überblicken kann. 
Aus den Reizungsversuchen des N. pharyng. med. am Hunde folgt:

1) Reizung des N. pharyng. med. erzeugt eine Contraction der gleichseitigen Pharynxmusculatur. Dadurch wird der ganze Kehlkopf nach unten und nach der Seite des gereizten Nerven gezogen. Eine Contraction des M. crico-thyreoid, erfolgt dabei nicht.

2) Besteht, wie es in einigen, aber seltenen Fällen vorkommt, zwischen dem äusseren Aste des N. laryng. sup. und dem N. pharyng. med, eine Anastomose, ohne dass der $\mathrm{N}$. pharyng. med. bis in den M. crico-thyreoid. zu verfolgen ist und reizt man nun den N. pharyng. oberhalb der Anastomose, so findet eine typische Contraction des ganzen M. crico-thyreoid. statt.

Durchschneidet man die Anastomose und reizt jetzt den N. pharyng. med., so contrahirt sich der M. crico-thyreoid. nicht mehr, sondern es findet blos noch eine Fältelung seiner äussersten, lateralen Partien statt; diese Fältelung wird erzeugt durch die Contraction der gleichseitigen M. constrictor pharyng. inf.

Exstirpirt man den ganzen N. laryng. sup. und reizt den N. pharyng. med., so findet keinerlei Contraction des M. cricothyreoid. statt; der Muskel wird vielmehr durch die sich contrahirenden Pharynxmuskeln nach unten und nach der Seite des gereizten Nerven gezogen.

Es besteht füglich beim Hunde keine Innervation des M. crico-thyreoid. seitens des N. pharyng. med. In den seltenen Fällen, wo bei Bestehen einer Communication zwischen Laryng. sup. and Pharyng. med. nach Reizung des N. pharyng. med. eine Contraction des M. crico-thyreoid. eintritt, ist letztere hervorgerufen dadurch, dass im N. pharyng. med. ausgeschaltete Fasern des N. laryng. sup. verlaufen.

In dritter Linie stellte ich Versuche bei der Katze an. An diesem Thiere ist der N. pharyng. med. und sein Verhalten zum M. crico-thyreoid. bisher überhaupt nicht untersucht.

Zunächst wurde der anatomische Verlauf des N. pharyng. med. festgestellt. Nach Freilegung der Trachea, des Larynx und Durchtrennung der Mm. sterno-hyoid. wurde der N. vagus bis zum For. jugulare freigelegt. Unterhalb des. Ganglion jugulare bezügl, am unteren Ende desselben entspringt der N. laryng. 
sup. Derselbe theilt sich erst ganz spät in einen äusseren und einen inneren Ast; der äussere Ast geht in den M. crico-thyreoid., der innere in der Mitte der Cart. thyreoid. durch dieselbe in den Larynx. Am oberen Ende des Ganglion jugulare nahe dem For. jugulare entspringt aus dem N. vagus der N. pharyng. vagi. Derselbe giebt alsbald nach seiner Entstehung eine Anastomose zum N. glossopharyng. und theilt sich alsdann in 2, selten in 3 Zweige; der obere geht in der Höhe der Membr. hyothyreoid. zur hochgelegenen Pharynxmusculatur; der untere, entsprechend dem N. pharyng. med. beim Hunde, verläuft auf der Pharynxmusculatur an der inneren Seite des N. laryng. sup. medial und abwärts, um in dem M. constrictor pharyng. inf. zu verschwinden. Ein Uebergang in den M. crico-thyreoid., sowie eine Anastomose mit dem N. laryng. sup. war nie nachzuweisen.

Bei der elektrischen Reizung des N. pharyng. med. contrahirt sich die ganze zugehörige Pharynxmusculatur; dabei wird der Kehlkopf und mit ihm der M. crico-thyreoid. nach der Seite des gereizten Nerven und nach unten gezogen. Eine Contraction des M. crico-thyreoid. tritt nicht ein.

20. April. Katze. Freilegung des linken N. vagus, N. laryng. sup. und N. pharyng. vagi. Der äussere Ast des N. laryng. sup. ist sehr fein. Der N. laryng. sup. geht in den Vagus unterhalb des Ganglion jngulare, der N. pharyng. vagi oberbalb desselben. Theilung des N. pharyng. vagi in zwei Aeste, Anastomose mit dem N. glossopharyng. Reizung des. N. laryng. sup. ergiebt eine typische Contraction des MI. crico-thyreoid. Bei Reizung des N. pharyng. med. contrahirt sich die ganze linke Pharynxrausculatur; der Larynx und mit ihm der M. crico-thyreoid. wird dabei nach links und unten gezogen. Eine Contraction des Muskels tritt nicht ein.

Die vorgenommene Präparation der rechten Seite ergiebt genau die oben ausgeführten Resultate. Auffallend ist, dass ausser den sonst vorhandenen, zwei Aesten des N. pharyng. vagi noch ein dritter sehr feiner Zweig in der Richtung des M. stylo-pbaryng. verläuft. Auch bestebt die Anastomose mit dem N. glossopharyng. Der N. laryng. sup. entspringt unterhalb, der N. pharyng. vagi oberhalb des Ganglion jugulare aus dem N. vagus. Der äussere Ast des N. laryng. sup. ist sebr fein.

25. April. Katze. Freilegung des N. laryng. sup. und N. pharyng, vagi sin. Reizung des N. laryng. sup. ergiebt Contraction des M. cricothyreoid. Bei Reizung des N. pharyng. med. contrahirt sich nur die gleichseitige Pharynxmusculatur. Dieselbe zieht dabei den Larynx nach links und untea. Eine Contraction des M. erico-thyreoid. wird bei Reizung des N. pharyng. med. nicht beobachtet. 
Die Präparation der rechten Seite ergiebt, dass der N. pharyng. vagi oberhalb des Ganglion jugulare ans dem N. vagus entspringt; eine Anastomose des N. pharyng. med. mit dem N. laryng. sup. oder ein Uebergang desselben in den $\mathbf{M}$. crico-thyreoid. ist nicht nachzuweisen.

27. April. Katze. Freilegung des linken N. laryng. sup. und N. pharyng. vagi. Reizung des N. laryng. sup.: Ausgiebige Contraction des M. cricothyreoid. Bei Reizung des N. pharyng. med. contrahirt sich die ganze gleichseitige Pharynxmusculatur und $z$ war so, dass dadurch der Larynx mit auf die linke Seite gezogen wird; eine Contraction der M. erico-thyreoid. findet bei Reizung des N. pharyng. med. nicht statt.

Die übrigen 7 vorgenommenen Yersuche an Katzen ergaben bei der Präparation und bei den Reizungsversuchen genau dasselbe Resultat, wie die nach den Protocollen wiedergegebenen. Ich führe dieselben deshalb hier nicht aus.

Es wurde nun an der Katze der Controlversuch gemacht wie beim Hunde: Der N. vagus wurde bis zum For. jugulare freigelegt, der N. laryng. sup. vollständig exstirpirt. Darauf wurde der N. vagus oberhalb der Abgangsstelle des $\mathrm{N}$. pharyng. vagi unterbunden und abgetrennt. Alsdann Reizung des M. vagus oberhalb der Abgangsstelle des $\mathrm{N}$. pharyng. vagi und dadurch Reizung des N. pharyng. med. e vago.

7. Juni. Katze. Freilegung des N. vagus dexter bis zum For. jugulare. Vollständige Exstirpation des $N$. laryng. sup. dexter. Unterbindung und Abtrennung des N. vagus dexter oberhalb der Abgangsstelle des N. pharyng. vagi. Reizung des N. vagus oberhalb der Abgangsstelle des $\mathrm{N}$. pharyng. vagi: Es erfolgt hierbei typische Contraction der ganzen recbtsseitigen Pharynxmuseulatur, keine des M. crico-thyreoid. dexter.

14. Juni. Katze. N. laryng. sup. ganz exstirpirt. N. vagus oberhalb des Ganglion jugulare unterbunden und abgetrennt. Bei Reizung vom N. vagus oberhalb der Abgangsstelle des N. pharyng. vagi keine Zuekung im rechten $\mathrm{M}$. crico-thyreoid.

22. Juni. Katze. Freilegung des linken N. vagus, N. pharyng. vagi, N. laryng. sup. Unterbindung und Abtrennung des $N$. vagus oberbalb der Abgangsstelle des N. pharyng. vagi. Exstirpation des ganzen N. laryng. sup. Bei Reizung des N. vagus aberhalb der Abgangsstelle des N. pharyng. vagi erfolgt nur Zuckung in den gleichseitigen Muskeln des Pharynx und öfters im Scbluckact, dagegen keine Zuckung im gleichseitigen M. cricothyreoid.

Aus den so angeordneten Versuchen folgt, dass der $\mathrm{N}$. pharyng. med. e vago bei der Katze keinen Znsammenhang mit dem M. crico-thyreoideus hat. Würde ein solcher zwischen dem 
genannten Nerven und Muskel bestehen, so müsste bei Reizung des $\mathrm{N}$. vagus oberhalb der $\mathrm{Abgangsstelle} \mathrm{des} \mathrm{N}$. pharyng. med. eine Contraction des M. crico-thyreoid. erfolgen.

Von Herrn Prof. Munk wurden mir zur Vervollständigung obiger Versuche zwei Affen überwiesen. Der anatomische Befund entsprach genau dem früher angegebenen. Der N. pharyng. vagi entspringt beim Affen aus dem $\mathrm{N}$. vagus zwischen $\mathrm{Pl}$. nodosus und dem For. jugulare. Er giebt alsbald eine Anastomose zum N. glossopharyng. $a b$ und erhält einen Verbindungszweig aus dem Ganglion supremum des Halssympathicus. Der Nerv theilt sich bald nach seiner Entstehung in zwei $Z_{\text {weige. Der }}$ obere verläuft in der Höhe der Membr. hyo-thyreoid. und geht in die Pharynxmusculatur, in der er verschwindet. Der untere Ast geht nach abwärts, liegt unterhalb des M. thyre0-pharyng., kreuzt den inneren Ast des N. laryng. sup., an dessen medialer Seite er liegt und verläuft dann wie der äussere Ast des N. laryng. sup. nach vorn und abwärts, um im M. constrictor pharyng. inf. zu verschwinden. Ein Uebergang des Nerven in den M. crico-thyreoid. besteht nicht.

5. Juli. Affe (Innus). Freilegung des linken N. vagus. Zuvor Unterbindung der V. jugular. ant., der Art. carotis comm., Durchsebneidung des N. glossopharyng. Diese Vornahmen sind nothwendig, da der Hals des Affen sehr kurz ist und der N. vagus an seiner Eintrittsstelle in das For. jugulare sebr tief liegt. Der N. pharyng. vagi theilt sich in zwei Zweigo, nachdem er je einen Verbindungszweig zum Ganglion supremum des Halssympathicus und zum N. glossopharyng. abgegeben bat. Unterbindung und Abtrennung des N. vagus oberbalb der Eintrittsstelle des N. pharyng. vagi. Exstirpation des ganzen N. laryng. sup. sin. Reizung des N. vagus oberhalb der Abgangsstelle des N. pharyng. vagi ergiebt Längsdehnung des M. crico-thyreoid. wie beim Hunde. Die Debnung des Muskels wird dadurch bervorgerufen, dass die Cart. cricoid. in Folge der Contraction der gesammten Pharynxmusculatur nach unten gezogen wird. Reizung des N. pharyng. med. nach seinem Abgange aus dem Vagus ergiebt Drehung des ganzen Keblkopfes und damit auch des M. erico-thyreoid. nach links und unten. Eine Contraction des M. crico-thyreoid. findet bei Reizung des N. pharyng. med. nicht statt.

Der am 10. Juli operirte zweite Affe stirbt in der Narkose.

Der eine Versuch beim Affen erlaubt kein völliges Endurtheil. In dem einen Falle contrahirte sich bei Reizung des N. pharyng. med. der gleichseitige M. crico-thyreoid. nicht. 
Die Beantwortung der Frage: Innervirt der N. pharyng. med. den M. crico-thyreoid.? stellt sich demnach so:

Reizung des N. pharyng. med. erzeugt

1) beim Kaninchen eine Contraction des M. crico-thyreoid.

2) beim Hunde in der Mehrzahl der Fälle keine Contraction des M. crico-thyreoid. In den seltenen Fällen, in denen eine Anastomose zwischen N. pharyng. med. und N. laryng. sup. besteht, wird bei Reizung des N. pharyng. med. eine Contraction des M. crico-thyreoid. hervorgerufen; dieselbe hört auf nach Durchschneidung der Anastomose.

3) bei der Katze und dem Affen keine Contraction des M. crico-thyreoid.

Es existirt somit nur beim Kaninchen, dagegen nicht beim Hunde, bei der Katze und beim Affen ein aus dem Ram. pharyng. vagi stammender Nerv, der den M. crico-thyreoid. motorisch innervirt. Herr Exner ist füglich berechtigt, einen N. laryng. med. beim Kaninchen, nicht berechtigt, beim Hunde, bei der Katze und beim Affen einen N. laryng. med. neben den wohlausgebildeten Nn. laryng. sup. und inf. anzunehmen. Die Abweichungen von der Norm, die bei letztgenannten Thieren vorkommen, lassen sich dahin zusammenfassen, dass in sehr wenigen Fällen Fasern des N. laryng. sup. den Vagus nicht im N. laryng. sup., sondern im N. pharyng. vagi verlassen und mittelst einer Anastomose zwischen dem N. pharyng. med. und dem N. laryng. sup. in den letzteren übergehen. 